\title{
Taking a disagreeing perspective improves the accuracy of people's quantitative
} estimates

This paper has been accepted for publication in Psychological Science

Philippe P. F. M. Van de Calseyde ${ }^{1+*}$, Emir Efendić $^{2} \dagger$

${ }^{1}$ Eindhoven University of Technology, Department of Industrial Engineering and Innovation Sciences, Human Performance Management Group, 5600 MB Eindhoven, The Netherlands;

${ }^{2}$ Maastricht University, School of Business and Economics, Marketing and Supply-Chain Management, Tongersestraat 53, 6211 LM, Maastricht, The Netherlands.

*Address correspondence to: Philippe Van de Calseyde, Eindhoven University of Technology, Department of Industrial Engineering and Innovation Sciences, Human Performance Management Group, 5600 MB Eindhoven, The Netherlands E-mail: P.P.F.M.v.d.Calseyde@tue.nl.

Orcid(s): https://orcid.org/0000-0003-0566-7018, https://orcid.org/0000-0002-2365-0247

\section{Keywords}

Disagreement; Perspective-Taking; Wisdom of Inner Crowds; Estimation; Accuracy

\section{Author contributions:}

$\dagger$ Both authors contributed equally to the work presented in this paper. Order of the author list was decided by a coin toss. 


\begin{abstract}
Many decisions rest upon people's ability to make estimates of unknown quantities. In these judgments, the aggregate estimate of a crowd of individuals is often more accurate than most individual estimates. Remarkably, similar principles apply when aggregating multiple estimates from the same person and a key challenge is to identify strategies that improve the accuracy of people's aggregate estimates. Here, we present the following strategy: combine people's first estimate with their second estimate made from the perspective of someone they often disagree with. In five pre-registered experiments $(N=6425$, with 53,086 estimates) with populations from the US and UK, we find that such a strategy produces accurate estimates (as compared to when people make a second guess, or when second estimates are made from the perspective of someone they often agree with). These results suggest that disagreement, often highlighted for its negative impact, is a powerful tool in producing accurate judgments.
\end{abstract}

\title{
Statement of relevance
}

In today's polarized society, disagreement is associated with conflict and division, but are there also benefits to disagreement? By utilizing people's ability to take the perspective of others, we propose that disagreement is a powerful tool in producing accurate estimates. In five experiments, people made estimates of unknown quantities from various perspectives. Following principles of within-person aggregation, we find that aggregating people's first estimate with their second estimate made from the perspective of someone they often disagree with produces accurate estimates. In explaining its accuracy, we find that taking a disagreeing perspective prompts people to consider estimates they normally would not consider as viable options, resulting in first- and second estimates that are more diverse and independent (and by extension more accurate when aggregated). Together, these results underscore the importance of perspective-taking and disagreement as strategies to improve the accuracy of people's quantitative estimates. 


\section{Introduction}

People often make estimates of some unknown quantities or events. In these types of judgments, a well-known phenomenon is that the average estimate of a crowd of individuals is often more accurate than most individual estimates (Galton, 1907; Surowiecki, 2005) and crowds have been used to improve judgments in areas such as economic forecasts (Clemen, 1989), medical diagnoses (Kurvers et al., 2016), weather forecasting (Baars \& Mass, 2005), and scientific research (Gordon et al., 2021; Altmejd et al., 2019).

The "wisdom of crowds" arises due to the (mathematical) principle whereby aggregating multiple imperfect, yet diverse, estimates diminishes the role of errors (Herzog \& Hertwig, 2009; Stroop, 1932). That is, when multiple estimates are sufficiently diverse and independent, averaging increases accuracy by canceling out errors across individuals (Vul \& Pashler, 2008). However, while beneficial, it is often not feasible for a single person to collect the estimates of multiple individuals. Remarkably, research suggests that the same principles underlying the wisdom of crowds also apply when aggregating multiple estimates from the same person - a phenomenon known as the "wisdom of the inner crowd" (Herzog \& Hertwig, 2009; Van Dolder \& Van den Assem, 2018; Vul \& Pashler, 2008).

It is not clear from the outset why aggregating multiple estimates from the same person would be beneficial. If a person's first estimate represents their best guess, then any other estimate would simply add noise (Hourihan \& Benjamin 2010; Vul \& Pashler, 2008). An alternative account based on probabilistic representations, however, argues that averaging estimates from the same person cancels out the errors that permeate people's judgments. Following this account, people's initial estimates represent samples drawn from an internal distribution of possible estimates, where second estimates are re-sampled guesses from that same distribution (Wallsten et al., 1997; Vul \& Pashler, 2008). When second, re-sampled 
estimates are sufficiently diverse, averaging increases accuracy by canceling out errors across estimates (Ariely et al., 2000; Herzog \& Hertwig, 2009; Keck \& Tang, 2020; Litvinova et al., 2020).

With such a powerful tool available to individuals, a key challenge is to identify strategies that can help improve the accuracy of people's aggregate estimates (Herzog \& Hertwig, 2014a). Research so far agrees that the inner crowd falters when people anchor too heavily on their first estimate when generating a second guess, thereby reducing diversity and independence (Herzog \& Hertwig, 2014a; Vul \& Pashler, 2008). To negate this, at least two methods have been applied. The first relies on the passage of time. For example, the benefits of aggregation tend to be higher with the introduction of time delays between both estimates (Steegen et al., 2014; Van Dolder \& Van den Assem, 2018; Vul \& Pashler, 2008). In these cases, the passage of time effectively de-anchors people from their first estimate (presumably through forgetting their initial estimate), thereby improving the diversity and independence of both estimates. A second method to increase diversity and independence is to rely on the mind's ability to construct alternative, opposing realities (Herzog \& Hertwig, 2009, 2014a, 2014b). A demonstrated way to do this has been through "dialectical bootstrapping" where people are prompted to base their second estimate on different assumptions and considerations (Herzog \& Hertwig, 2009). These dialectical estimates ideally result in errors with different signs relative to first estimates and there are different techniques to elicit such dialectical estimates. One technique, based on the "consider-the-opposite" strategy (Lord et al., 1984), instructs people to actively question the accuracy of their first estimate when generating a second guess. This technique has been shown to increase the accuracy of people's aggregate estimate by getting the same person to generate first- and second estimates that are more diverse and independent (Herzog \& Hertwig, 2009; 2014b). In this paper, we similarly rely on the mind's ability to construct opposing realities by prompting 
people to complement their initial estimate with a second estimate made from the perspective of someone they often disagree with.

Perspective-taking refers to people's ability to consider situations and events from the viewpoint of others (Piaget, 1965/1932). It has been associated with many positive outcomes such as altruistic behaviors, decreased stereotype expressions, and increased creativity (Batson et al., 1997; Galinsky \& Moskowitz, 2000; Hoever et al., 2012). However, following the principles of within-person aggregation, simply getting people to take the perspective of others would not be enough. What is needed is to add an estimate from the perspective of someone whose views are substantially different - in other words, to create a diverse inner crowd. To do this, we suggest using an oft-encountered component of people's interaction with others - disagreement. More specifically, as a viable method to obtain more diverse estimates, we propose to combine people's initial estimate with their second estimate made from the perspective of someone they often disagree with.

Disagreement is often decried as an undesirable component of people's interactions with others. In today's polarized society, disagreement has been associated with conflict, division, and misinformation (Kennedy \& Pronin, 2008; Reeder et al., 2005; Sunstein, 2002). However, although generally undesirable, research in group decision-making indicates that disagreement may actually be beneficial when groups address complex problems such as making estimates of unknown quantities or events (de Oliveira \& Nisbett, 2018; Hong \& Page, 2004; Mutz, 2006; Page, 2008). These effects occur due to the notion that disagreeing individuals tend to produce more diverse estimates, and by extension errors, which are cancelled out across group members when averaged (Page, 2008). It is precisely this aspect of disagreement that we rely on in our pursuit to foster more diverse estimates from the same individual. More specifically, we surmise that just as disagreement between different 
individuals is beneficial for the wisdom of crowds, so too, through perspective-taking, will this be beneficial for the wisdom of the inner crowd.

In understanding its benefits, we propose and test (in Experiment 3) that taking a disagreeing perspective leads to two distinct observations. First, from a disagreeing perspective, people are more likely to consider estimates that are strikingly different than their own guesses, thereby opening the sampling space of possible second estimates. And second, people are more likely to adopt these different estimates as their second estimates when viewing problems from a disagreeing perspective, leading to first- and second estimates that are more diverse and independent. These conjectures follow from earlier work on anchoring showing that people typically avoid making second estimates that are strikingly different from prior estimates or anchors (Epley \& Gilovich, 2006; Lewis et al., 2019; Tversky \& Kahneman, 1992). Making an estimate from a disagreeing perspective is expected to attenuate this tendency given that disagreeing others (almost by default) consider and adopt entirely different estimates as one's own estimate. However, although generally beneficial, the final experiment (Experiment 4) identifies a situation where taking a disagreeing-perspective backfires, undermining the benefit of averaging (i.e., in situations where second estimates are likely to be made in the wrong direction).

\section{Experiments 1a and 1b}

For easier viewing, we first present general methodological information that concerns all five experiments before describing the methods of Experiments 1a and 1b. Ethical approval for all experiments was obtained from the ethical review board at TU/e (Eindhoven University of Technology) with reference number: ERB2020IEIS29. For all studies, we report how we determined the sample size, all data exclusions (if any), all manipulations, and all measures. Data, code, and materials are publicly available at https://osf.io/qsxp8/. For analysis, we used mixed-effect models allowing us to make more generalizable claims across 
a wide range of participants and questions by employing random intercepts for participants and questions (Judd et al., 2012). We fitted the models using lme4 (Bates et al., 2015) and produced p-values using the Satterthwaite approximations for degrees of freedom from lmerTest (Kuznetsova et al., 2017). As there is little agreed upon definition on how to calculate effect sizes for mixed models, we report classic Cohen's $d$ or $d z$ effects calculated from the t-values of the fixed effect results obtained in the models (Cohen, 1988). For comparison of correlation coefficients between experimental conditions, we took a two-step approach. As participants responded to multiple questions twice, we first calculated the correlation between the errors of first- and second estimates (i.e., true answer subtracted from an estimate $^{1}$ ) for each participant. We then compared these Pearson's $r$ values across experimental conditions using independent-samples t-tests and report Cohen's $d$ effect sizes. All experiments' hypotheses, designs, and main analyses were pre-registered ${ }^{2}$ - Experiment 1a: https://osf.io/8hkjg; Experiment 1b: https://osf.io/kxa6w; Experiment 2: https://osf.io/pnqy2; Experiment 3: https://osf.io/7nh9z; Experiment 4 second-wave: https://osf.io/6mp98/. Sample size estimation for all experiments was based on a-priori power analyses using $\mathrm{G}^{*}$ Power, designed to have, for Experiments 1a, 1b, 2, and 3: $99 \%$ power to detect an effect size of Cohen's $d=.30$ (410 per condition), and $>80 \%$ power for an effect of Cohen's $d=.20$ (394 per condition); for Experiment 4: 95\% power to detect an effect size of Cohen's $d=.30$ (290 per condition), 80\% power for Cohen's $d=.25$ (253 per condition). Alpha was set at .05. For all experiments, we stopped data collection once we reached the predetermined sample sizes. Following previous studies on the inner crowd, to verify the

\footnotetext{
${ }^{1}$ Here, we kept the sign of the error as the size as well as the direction of the error are informative.

${ }^{2}$ Following prior work on the inner crowd, we initially pre-registered conducting simple t-tests rather than mixed-effect models for Experiments 1a, 2, and 4. We made the change in response to a suggestion during the review process.
} 
accuracy of people's estimates, we relied on the mean squared error (MSE) $)^{3}$ obtained by squaring the subtraction of the true answers from the estimations and then averaging.

\section{Method}

Design and procedure. In Experiment 1a participants made two weight estimates of 10 objects shown on pictures (see Table S1 in the Supplemental Online Material, reviewed; SOM-R for a list of the questions used in all experiments). In Experiment $1 \mathrm{~b}$ we used a different estimation task: participants made two estimates of 6 questions whose answer was in the $0 \%-100 \%$ range. The questions' true answers were obtained from various online sources (e.g., Wikipedia for Experiment 1a and the CIA world factbook for Experiment 1b). We collected data on Mturk (Experiment 1a: 880 participants from the US, Med $_{\text {Age }}=36$, $I Q R_{\text {Age }}=16 ; 51 \%$ female) and Prolific (Experiment 1b: 894 participants from the UK, $M e d_{\text {Age }}=35, I Q R_{\text {Age }}=20 ; 69 \%$ female $)$. After making their first estimate for all questions, half of the participants were told to make a second guess while the other half was instructed to make their second estimate from the perspective of a friend they often disagree with.

Participants were instructed not to look up the true answers during the study. They were randomly presented with the questions in two estimation stages (histograms for the distribution of participants' answers on both estimates for all five experiments can be accessed here: https://osf.io/q3tfh/). Participants were not told at the onset that they would be asked to make an additional, second estimate. In the first stage, participants simply provided their own estimates to the questions. The instructions for the second estimation stage were different depending on the condition. For the self-perspective condition, participants were told: "We will now ask you to provide a second guess at the answer to each of the ten (six; Experiment 1b) questions you were asked in the first session. These answers should not be the

\footnotetext{
${ }^{3}$ Using the mean absolute error produces the same results qualitatively. MAE results for all experiments can be found here: https://osf.io/ewpyq/
} 
same as your previous answers: these should reflect your 'second guess'." For the disagreeing perspective condition, participants were told: "Now picture a friend whose views and opinions are very different from yours. To illustrate, when discussing politics, you often find yourself disagreeing on various issues. How would he or she answer these ten (six; Experiment $1 b)$ questions? Please answer these questions again, but now as this friend." After responding to the questions, participants were asked to provide their age and gender. In addition, they were presented with an instructional manipulation check question instructing them to choose a particular option in a multiple-choice array and a question asking them whether they looked up any of the answers to the questions. Following the pre-registration plans, those who failed the instructional check and those who said they looked up the answers were excluded.

\section{Results}

Correlations. Comparing the two experimental conditions, the results showed that our instructions led to lower correlations when second estimates were made from a disagreeing perspective (1a: Mean $\mathrm{r}_{\text {disagree }}=.54 \mathrm{vs}$. Mean $\mathrm{r}_{\text {self }}=.71 ; 1 \mathrm{~b}$ : Mean $\mathrm{r}_{\text {disagree }}=.34 \mathrm{vs}$. Mean $\mathrm{r}_{\text {self }}=.73$ ). In both experiments, these two correlation coefficients were significantly different (1a: $d=.44 ; 1 \mathrm{~b}: d=.98$, both ps. $<.001)$, indicating that participants in the disagreeing-perspective condition produced more diverse estimates and errors as compared to participants in the self-perspective condition (see also Fig. 1a and 1b - scatterplots for each question separately for all five experiments can be accessed here: https://osf.io/q3tfh/).

[Figure 1 about here] 
a

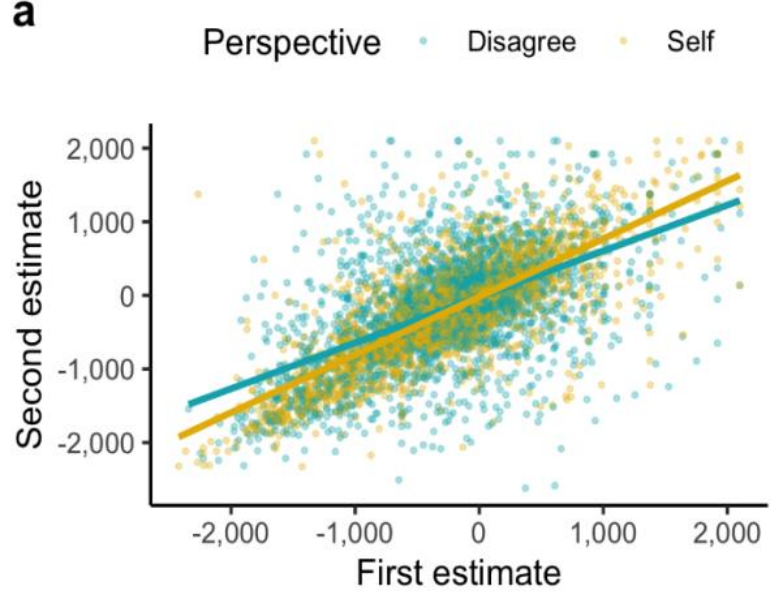

C

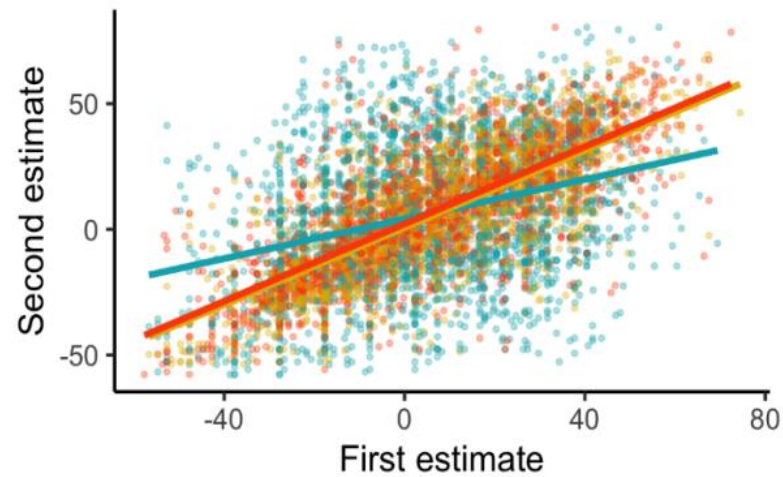

e

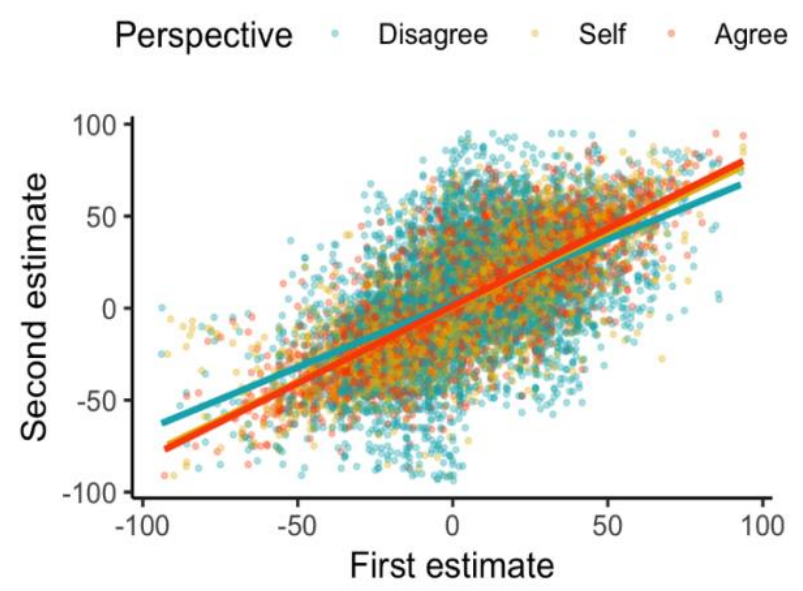

Figure 1. Diversity of errors across all five experiments. Signed errors indicate that for each experiment $(\mathrm{a}=$ Experiment $1 \mathrm{a}, \mathrm{b}=$ Experiment $1 \mathrm{~b}, \mathrm{c}=$ Experiment $2, \mathrm{~d}=$ Experiment 3 , and $\mathrm{e}=$ Experiment 4), the correlation between the estimates' errors was lower in the disagreeing-perspective (in green) than in the self-perspective (in yellow) and the agreeing-perspective (in red) conditions, indicating more error diversity. Each dot represents the error of a person's first- and second estimate in response to a particular question. b Perspective Disagree Self

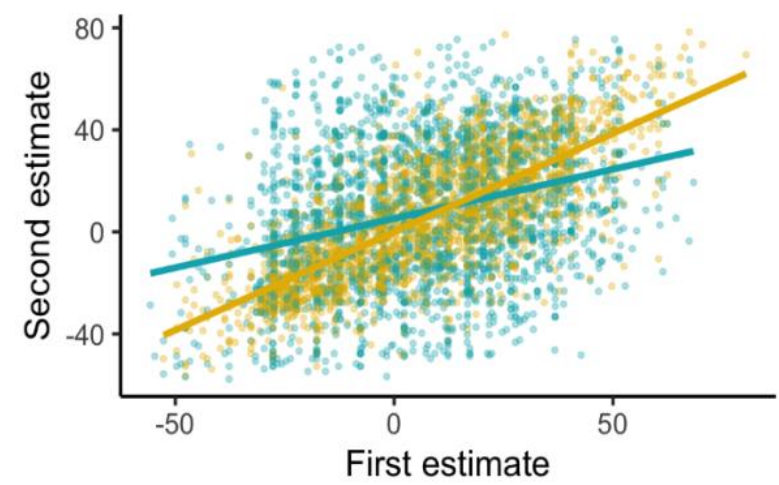

d Perspective Disagree Self . Agree

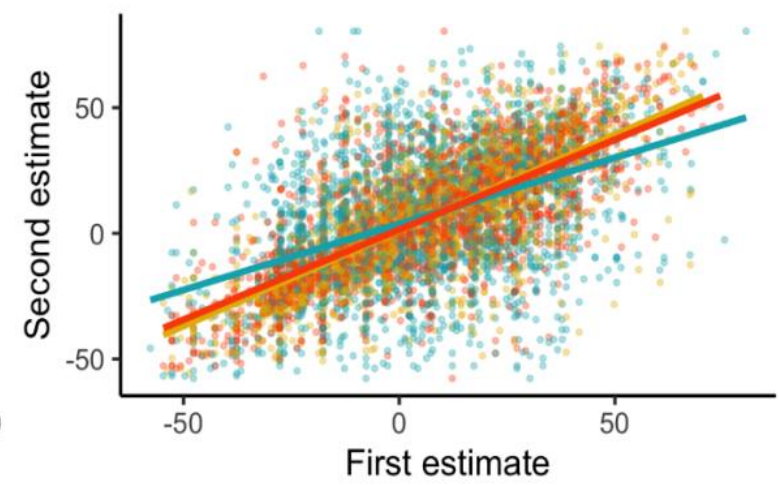

First estimate 
Inner crowd effects. For the inner crowd to be more accurate, the aggregate of both estimates should have a lower error than a person's first estimate alone. Taking into account both conditions (overall) and looking at the self- and disagree conditions separately, we found an inner crowd effect in Experiments $1 \mathrm{a}$ and $1 \mathrm{~b}$ (see Table 1 for summary statistics). The average of both estimates had a lower MSE than the first (and second) estimate alone, respectively (see Tables S2a and S2b in the SOM-R for descriptive statistics).

[Insert Table 1 about here]

Table 1. Inner crowd effect for Experiments 1a and 1b: comparing the average of two estimates with the first- and second estimate. Effect sizes $d_{\mathrm{z}}$ and $p$ values are shown.

\begin{tabular}{llccccccc}
\hline & & \multicolumn{2}{c}{ Overall } & \multicolumn{2}{c}{ Disagree } & \multicolumn{2}{c}{ Self } \\
\cline { 3 - 8 } & & $d_{z}$ & $p$ & $d_{z}$ & $p$ & $d_{z}$ & $p$ \\
\cline { 3 - 8 } & & .19 & $<.001$ & .23 & $<.001$ & .15 & .002 \\
\multirow{2}{*}{ Ex 1a } & First est. vs. Average & Second est. vs. Average & .28 & $<.001$ & .40 & $<.001$ & .16 & .001 \\
& Eirst est. vs. Average & .22 & $<.001$ & .28 & $<.001$ & .16 & $<.001$ \\
& \multirow{2}{*}{ Ex 1b } & Second est. vs. Average & .53 & $<.001$ & .79 & $<.001$ & .24 & $<.001$ \\
\hline \hline
\end{tabular}

Benefit of averaging. Would participants in the disagreeing-perspective condition benefit more from averaging their estimates than participants in the self-perspective condition? To test this, we calculated the benefit of averaging by subtracting the squared error of average estimates from the squared error of first estimates (similar procedures have been used before - Herzog \& Hertwig, 2009; Steegen et al., 2014; Vul \& Pashler, 2008). The higher this number, the larger the benefit of averaging (i.e., the more accurate the inner crowd). Overall, the results indicate that in both Experiment $1 \mathrm{a}$ and $1 \mathrm{~b}^{4}$ participants in the

\footnotetext{
${ }^{4}$ Note that in Experiment $1 \mathrm{~b}$ we also measured the time participants needed to generate their second estimates. Comparing time between the self- and the disagreeing-perspective conditions showed that there was no difference, $d=.08, p=.21$, with a $\mathrm{BF}_{01}=8.78$.
} 
disagreeing-perspective condition indeed benefited more from averaging their estimates than participants in the self-perspective condition (1a: $d=.16, p=.02 ; 1 \mathrm{~b}: d=.18, p=.01)$.

Bracketing. To more concretely test whether people in the disagreeing-perspective condition benefited more from averaging, we looked at bracketing rates across conditions. Bracketing is a key component underpinning the benefit of aggregating multiple estimates (Larrick \& Soll, 2006). It refers to the observation that if two estimates are on the opposite sides of the true answer, thus bracketing it (i.e., one overestimating the true answer while the other underestimates it), aggregating them will typically result in a more accurate average estimate (Larrick \& Soll, 2006; Soll \& Larrick, 2009). As such, for each question, we verified whether the question's true answer was bracketed by the two estimates. As expected, the bracketing rate was much higher in the disagreeing-perspective condition at $29 \%$ (Experiment 1a) and at 38\% (Experiment 1b), compared to the self-perspective condition where 19\% (Experiment 1a) and 20\% (Experiment 1b) of people's estimates bracketed the questions' true answers (1a: $d=.56, p<.001 ; 1 \mathrm{~b}: d=.80, p<.001)$.

\section{Experiment 2}

People who made a second estimate from the perspective of a person they often disagree with benefited more from averaging than people who simply made a second guess. The following experiment provides an important extension. Specifically, we included a third experimental condition in which participants were instructed to take the perspective of a person they often agree with. We included this condition to underscore the need to take a disagreeing perspective to improve the accuracy of people's inner crowds.

\section{Method}

Design and procedure. The procedure of this experiment was similar to Experiment 1b. However, we added an additional condition (i.e., the agreeing perspective condition) where the instructions for the second estimation stage were: "Now picture a friend whose 
views and opinions are very similar to yours. To illustrate, when discussing politics, you often find yourself agreeing on various issues. How would he or she answer these six questions? Please answer these questions again, but now as this friend." We gathered data of 1389 participants from the US on Mturk $\left(M e d_{\text {Age }}=35, I Q R_{\text {Age }}=16 ; 44 \%\right.$ female $)$.

\section{Results}

Correlations. The estimates' errors were highly correlated in the self-perspective and the agreeing-perspective conditions (Mean $r_{\text {self }}=.73$ vs. Mean $r_{\text {agree }}=.74$ ). This correlation was much lower in the disagreeing-perspective condition (Mean $r_{\text {disagree }}=.32$ ) (see also Fig. 1c). Comparing these correlation coefficients, participants in the disagreeing-perspective condition produced more diverse errors than both participants in the self- $(d=.99)$ or agreeing-perspective $(d=1.00)$ conditions (both $p s<.001)$, while there was no difference in the diversity of errors between the self- and agree-perspective conditions $(d=.02 ; p=.81)$.

Inner crowd effects. There was again an inner crowd effect overall (taking into account all three conditions) and in the three conditions separately (see top panels in Table 2 and see Table S3 in the SOM-R for descriptive statistics).

[Insert Table 2 about here]

Table 2. Inner crowd effects for Experiments 2 and 3: comparing the average of two estimates with the first- and second estimate. Effect sizes $d_{\mathrm{z}}$ and $p$ values are shown.

\begin{tabular}{|c|c|c|c|c|c|c|c|c|c|}
\hline & & \multicolumn{2}{|c|}{ Overall } & \multicolumn{2}{|c|}{ Disagree } & \multicolumn{2}{|c|}{ Self } & \multicolumn{2}{|c|}{ Agree } \\
\hline & & $d z$ & $p$ & $d z$ & $p$ & $d z$ & $p$ & $d z$ & $p$ \\
\hline & First est. vs. Average & .19 & $<.001$ & .28 & $<.001$ & .15 & .001 & .14 & .003 \\
\hline Ex 2 & $\begin{array}{l}\text { Second est. vs. } \\
\text { Average }\end{array}$ & .44 & $<.001$ & .80 & $<.001$ & .25 & $<.001$ & .23 & $<.001$ \\
\hline & First est. vs. Average & .21 & $<.001$ & .25 & $<.001$ & .17 & $<.001$ & .23 & $<.001$ \\
\hline Ex 3 & $\begin{array}{l}\text { Second est. vs. } \\
\text { Average }\end{array}$ & .33 & $<.001$ & .56 & $<.001$ & .22 & $<.001$ & .19 & $<.001$ \\
\hline
\end{tabular}


Benefit of averaging. No difference between the self- and agreeing-perspective conditions with regards to benefit of averaging $(d=.03, p=.61)$. Importantly, participants in the disagreeing-perspective condition again benefited more from averaging both estimates as compared to the self- and agreeing perspective conditions $(d=.18, p=.01$ and $d=.21, p=$ .001 , respectively).

Bracketing. With $21 \%$ and $20 \%$ of people's estimates bracketing the questions' true answers, there was no difference in bracketing rates between the self- and agreeingperspective conditions $(d=.08, p=.25)$. Crucially, however, the bracketing rate was again higher in the disagreeing-perspective condition with $39 \%$ of people's estimates bracketing the questions' true answers, compared to both the self- $(d=.85, p<.001)$ and the agreeingperspective conditions $(d=.90, p<.001)$.

\section{Experiment 3}

In Experiment 3, we tested the proposed mechanism explaining the observation of more diversity and independence when second estimates are made from a disagreeing perspective. Earlier work on inner crowds suggest that people typically anchor too heavily on first estimates when generating a second guess, thereby not producing diverse enough estimates and errors (Herzog \& Hertwig, 2009; Vul \& Pashler, 2008). Making an estimate from a disagreeing perspective was expected to attenuate this tendency given that disagreeing others (almost by default) consider and adopt entirely different estimates as one's own estimate.

\section{Method}

Design and procedure. The design and procedure was similar to Experiment 2. However, before making their second estimate, participants in the self-perspective condition were asked: "What is the most extreme estimate (either extremely high or extremely low) that you would consider as second guess to this question?". In the agree- and disagree-perspective 
conditions participants were asked: "What is the most extreme estimate (either extremely high or extremely low) that your friend would consider as answer to this question?". We gathered data from 1426 participants from the US on Mturk $\left(M e d_{\text {Age }}=36, I Q R\right.$ Age $=17 ; 48 \%$ female $)$.

\section{Results}

Correlations. The estimates' errors were again highly correlated in the selfperspective and the agreeing-perspective conditions (Mean $r_{\text {self }}=.74$ vs. Mean $r_{\text {agree }}=.72$ ). This correlation was much lower in the disagreeing-perspective condition (Mean $r_{\text {disagree }}=$ .46) (see also Fig. 1d). Comparing these correlation coefficients, participants in the disagreeing-perspective condition again produced more diverse errors than both participants in the self- $(d=.78)$ or agreeing-perspective $(d=.71)$ conditions (both $p s<.001)$, while there was no difference in error diversity between the self- and agree-perspective conditions $(d=$ $.07 ; p=.26)$

Inner crowd effects. There was an inner crowd effect overall (taking into account all three conditions) and in the three conditions separately (see lower panels in Table 2 and Table S4 in SOM-R for descriptive statistics).

Benefit of averaging. Agree was slightly better than self $(d=.13, p=.04)$. Importantly, participants in the disagreeing-perspective condition again benefited more from averaging both estimates as compared to the self-perspective condition $(d=.15, p=.02)$. However, there was no difference between the agreeing- and disagreeing-perspective conditions $(d=.04, p=.55)^{5}$ although descriptively, the effect was in the right direction.

Bracketing. With $20 \%$ and $21 \%$ of people's estimates bracketing the questions' true answers, there was no difference in bracketing rates between the self- and agreeing-

\footnotetext{
${ }^{5}$ When performing multiple studies, the presence of some non-significant findings is to be expected given the nature of hypothesis testing (Lakens \& Etz, 2017). To assess the overall evidential value of the prediction that the benefit of averaging is higher when taking a disagreeing perspective, we aggregated the data of the same 6 questions of Experiments 2, 3, and 4. Results show that, overall, participants in the disagreeing-perspective condition benefited more from averaging than participants in the agreeing-perspective condition $(d=.18, p<$ $.001)$.
} 
perspective conditions $(d=.05, p=.42)$. Crucially, however, the bracketing rate was again higher in the disagreeing-perspective condition with $33 \%$ of people's estimates bracketing the questions' true answers, compared to both the self- $(d=.64, p<.001)$ and the agreeingperspective conditions $(d=.61, p<.001)$.

Extreme estimate analysis. To test the proposition that taking a disagreeing perspective prompts people to consider more extreme estimates as possible answer to a question, we computed the (absolute) difference score between a person's first estimate and the most extreme estimate that they (or their friend) would consider as answer. As expected, there was no difference between participants in the agreeing- and self-perspective conditions $(d=.04, p=.51)$. Importantly however, participants in the disagreeing-perspective condition considered far more extreme estimates as possible answer than either participants in the self$(d=.41, p<.001)$ or the agreeing-perspective conditions $(d=.46, p<.001)$. Moreover, to test whether participants in the disagreeing-perspective condition would also be more inclined to adopt this extreme estimate as second answers, we computed the (absolute) difference score between a person's second estimate and the most extreme estimate. The lower this number, the closer the second estimate to the most extreme estimate. As expected, there was no difference between participants in the agreeing- and self-perspective conditions $(d=.12, p=.06)$. Importantly, participants in the disagreeing-perspective condition made second estimates much closer to the extreme estimate than either the participants in the self$(d=.29, p<.001)$ or the agreeing-perspective conditions $(d=.14, p=.03)$ (see SOM-R Table S5 for descriptive statistics). The willingness of participants to adopt these extreme estimates as answer is noteworthy, given people's general propensity to avoid making extreme judgments (Lewis et al., 2019). This aversion seems to dissipate when second estimates are made from the viewpoint of disagreeing others. Interestingly, even if people made second estimates equally close to their most extreme estimate from a disagreeing 
perspective, they would still produce more diverse estimates given that these extreme estimates are generally more extreme. Overall, these results underscore the conjecture that taking a disagreeing perspective prompts people to consider and adopt second estimates that are strikingly different than their initial estimate, rendering a set of estimates that is more diverse and independent.

\section{Experiment 4}

The final experiment identifies a situation where taking a disagreeing-perspective backfires. Specifically, this was expected in situations where a question's true answer lies close to the (lower- or upper-) end of a scale. (e.g., the true answer is $2 \%$ or $98 \%$ on a $0 \%$ $100 \%$ scale) and when a person's initial estimate is close to this answer. For example, imagine being asked the following question: "What percent of China's population identifies as Christian?". The true answer to this question is $5.1 \%$ and if you are like most people, your first estimate is probably leaning towards this lower end of the scale (say your first estimate is $10 \%)$. Given the position of the question's true answer and your first estimate, your second estimate is likely (in general) to move away from the answer towards the opposite side of the scale (Juslin et al., 2000), effectively hurting the accuracy of your average estimate. Importantly, such a movement is expected to be especially harmful when second estimates are made from a disagreeing-perspective as now, given the propensity to adopt more extreme estimates from such a perspective (see Experiment 3), these estimates move away from the true answer to a much greater extent (resulting in an average estimate that is far worse than the initial, first estimate).

\section{Method}

Design and procedure. We gathered data in two waves. We preregistered our hypotheses and analysis plan for the second wave. Since the procedures in the two waves were identical, we decided to combine them (analyzing the data separately yields similar 
results and can be accessed here: https://osf.io/ewpyq/). The procedure of this experiment was similar to Experiment 2 in all but two respects. First, we added an additional 6 questions thus ending up with 12 questions in total. Second, we categorized the questions according to where the true answer fell, i.e., whether the true answer lied in the mid-scale or end-scale (0\%-10\% or $90 \%-100 \%)$. Participants thus made two estimates about a set of 12 questions, all of which had a true answer that was in the $0 \%$ to $100 \%$ range. Crucially, half of the questions' true answers were close to the (lower- or upper-) end of the scale, from 0\%-10\% and $90 \%-100 \%$. For the other half of the questions, true answers were relatively far from the end of the scale (e.g., 58\%). Combining the two data wave collections resulted in a sample of 1836 US participants from Mturk $\left(\operatorname{Med}_{\text {Age }}=36, I Q R\right.$ Age $=17 ; 51 \%$ female $)$.

\section{Results}

Correlations. Correlations between the estimates' errors in the self- and agreeingperspective conditions were again high (Mean $r_{\text {self }}=.77$, Mean $\left.r_{\text {agree }}=.79\right)$. This correlation was lower in the disagreeing-perspective condition (Mean $r_{\text {disagree }}=.57$ ) (see also Fig. 1e). Participants in the disagreeing-perspective condition produced more diverse errors than both participants in the self- $(d=.83)$ or agreeing-perspective $(d=.96)$ conditions (both $p s<$ $.001)$. The difference between the self- and agree-perspective was also significant $(d=.15, p$ $=.01)$. Overall, the disagreeing-perspective condition again produced more diverse errors.

Inner crowd effects. Taking into account both types of questions (mid-scale and the end-scale questions) and all three conditions, we did not find an inner crowd effect (see Table 3). Importantly, and in line with our proposal, the perspective-taking instructions had a markedly different impact when looking at the mid-scale and the end-scale questions separately. For the mid-scale questions, there was an inner crowd effect similar to the previous experiments. However, when looking at the end-scale questions for the disagreeing- 
perspective condition, the average of both estimates had a much higher error than the first estimate alone (see Table S6 in the SOM-R for descriptives).

[Insert Table 3 around here]

Table 3. Inner crowd effect for Experiment 4: comparing the average of two estimates with the first- and second estimate. Effect sizes $d_{\mathrm{z}}$ and $p$ values are shown across both question types and separately.

\section{Both question types}

\begin{tabular}{ccccccccc}
\hline \multicolumn{2}{c}{ Overall } & \multicolumn{2}{c}{ Disagree } & \multicolumn{2}{c}{ Self } & \multicolumn{2}{c}{ Agree } \\
\hline$d_{z}$ & $p$ & $d_{z}$ & $p$ & $d_{z}$ & $p$ & $d_{z}$ & $p$ \\
\hline .03 & .16 & .05 & .20 & .08 & .05 & .08 & .06 \\
& & & & & & & \\
.54 & $<.001$ & .88 & $<.001$ & .36 & $<.001$ & .29 & $<.001$
\end{tabular}

Second est. vs.

Average

First est. vs.

Average

54

Mid-scale questions

\begin{tabular}{llllllllll}
\hline \multicolumn{2}{c}{ Overall } & & \multicolumn{2}{c}{ Disagree } & & \multicolumn{2}{c}{ Self } & \multicolumn{2}{c}{ Agree } \\
\hline$d_{z}$ & $p$ & $d_{z}$ & $p$ & $d_{z}$ & $p$ & $d_{z}$ & $p$ \\
\hline
\end{tabular}

First est. vs.

Average

$\begin{array}{llllllll}.19 & <.001 & .28 & <.001 & .14 & .001 & .15 & <.001\end{array}$

Second est. vs.

Average

$<.001 \quad .70$

$<.001$

$.34<.001$

$.26<.001$

\section{End-scale questions}

\begin{tabular}{ccccccccc}
\hline \multicolumn{2}{c}{ Overall } & \multicolumn{2}{c}{ Disagree } & \multicolumn{2}{c}{ Self } & \multicolumn{2}{c}{ Agree } \\
\hline$d_{z}$ & $p$ & $d_{z}$ & $p$ & $d_{z}$ & $p$ & $d_{z}$ & $p$ \\
\hline-.09 & $<.001$ & -.26 & $<.001$ & .01 & .90 & .01 & .71 \\
& & & & & & & \\
.38 & $<.001$ & .66 & $<.001$ & .21 & $<.001$ & .19 & $<.001$
\end{tabular}

Benefit of averaging. For the mid-scale questions, we found no difference between the self- and agreeing-perspective conditions $(d=.05, p=.39)$ while the benefit of averaging 
was again higher for participants in the disagreeing-perspective condition, compared to the self- $(d=.28, p<.001)$ and agreeing-perspective condition $(d=.24, p<.001)$. Thus, for the mid-scale questions, the results echo those obtained in the previous experiments. For the endscale questions, there was no difference between the self- and agreeing-perspective conditions $(d=.05, p=.39)$. However, in the disagreeing-perspective condition, averaging was actually much more disadvantageous than in the self- $(d=-.41, p<.001)$ and agreeingperspective conditions $(d=-.40, p<.001)$.

Bracketing. For the mid-scale questions, with $23 \%$ and $21 \%$ of the estimates bracketing the questions' true answers there was slightly more bracketing in the self-, compared to the agreeing-perspective condition $(d=.13, p=.03)$. Importantly, as expected, the degree of bracketing was again higher in the disagreeing-perspective condition where $37 \%$ of the estimates bracketed the questions' true answers, compared to both the self- $(d=$ $.65, p<.001)$ and agreeing-perspective conditions $(d=.78, p<.001)$.

Focusing on the end-scale questions, we see generally lower rates of bracketing. With $13 \%$ and $11 \%$ of the estimates bracketing the questions' true answers, there was slightly more bracketing in the self-, compared to the agreeing-perspective condition $(d=.15, p=.01)$. The degree of bracketing was again higher in the disagreeing-perspective condition where $19 \%$ of estimates bracketed the questions' true answers, compared to both the self- $(d=.34, p<$ $.001)$ and agreeing-perspective conditions $(d=.48, p<.001)$.

\section{Understanding averaging and bracketing - when is it (un)beneficial. Prior} research suggests that bracketing is a key component in understanding why averaging estimates renders an improvement (Larrick \& Soll, 2006; Soll \& Larrick, 2009). However, as demonstrated by our results on the end-scale questions, this may not always be the case. Specifically, while we observed higher rates of bracketing in the disagreeing-perspective condition for end-scale questions, averaging nonetheless led to more overall disbenefit in this 


\section{DISAGREEING PERSPECTIVES AND ESTIMATION ACCURACY}

condition. To better understand why this occurred, we took a closer look at the underlying components that determine whether averaging first- and second estimates is beneficial or not.

We formalize each component in Equation 1.

$$
\frac{1}{n} \sum_{i \in n}\left(E_{i}^{X^{\mathrm{f}}}-E_{i}^{X^{\mathrm{a}}}\right)=\frac{1}{n}\left[\sum_{i \in n^{1}}\left(E_{i}^{X^{\mathrm{f}}}-E_{i}^{X^{\mathrm{a}}}\right)+\sum_{i \in n^{2}}\left(E_{i}^{X^{\mathrm{f}}}-E_{i}^{X^{\mathrm{a}}}\right)+\sum_{i \in n^{3}}\left(E_{i}^{X^{\mathrm{f}}}-E_{i}^{X^{\mathrm{a}}}\right)+\sum_{i \in n^{4}}\left(E_{i}^{X^{\mathrm{f}}}-E_{i}^{X^{\mathrm{a}}}\right)\right]
$$

where:

$i=$ index for individuals

$E_{i}^{X^{\mathrm{f}}}=$ error of the first estimate $\left(X^{\mathrm{f}}\right)$ of an $\mathrm{i}$-th individual on a particular question

$E_{i}^{X^{\mathrm{a}}}=$ error of the average estimate $\left(X^{\mathrm{a}}\right)$ of an i-th individual on a particular question

$n=$ total set of observations (i.e., number of individuals times the number of questions)

$n^{1}=$ subset of observations in $n$ where the second estimate $\left(X^{s}\right)$ moves towards the true answer $(X)$ while $X^{\mathrm{f}}>X^{\mathrm{s}}>X^{\mathrm{f}}-4\left(X^{\mathrm{f}}-X\right)$ or $X^{\mathrm{f}}<X^{\mathrm{s}}<X^{\mathrm{f}}+4\left(X-X^{\mathrm{f}}\right)$

$n^{2}=$ subset of observations in $n$ where $X^{\mathrm{s}}$ moves away from $X$ (i.e., $X<X^{\mathrm{f}}<X^{\mathrm{s}}$ or $X>X^{\mathrm{f}}>X^{\mathrm{s}}$ )

$n^{3}=$ subset of observations in $n$ where $X^{\mathrm{s}}$ moves towards $X$ while $X^{\mathrm{f}}>X^{\mathrm{s}}<X^{\mathrm{f}}-4\left(X^{\mathrm{f}}-\right.$ $X)$ or $X^{\mathrm{f}}<X^{\mathrm{s}}>X^{\mathrm{f}}+4\left(X-X^{\mathrm{f}}\right)$.

$n^{4} \quad=$ subset of observations in $n$ where $X^{\mathrm{f}}=X$ while $X^{\mathrm{s}} \neq X^{\mathrm{f}}$

To clarify, the left-hand side of the equation represents the benefit of averaging while the right-hand side represents the unique components that make up this benefit of averaging. The first component $\left(n^{1}\right)$ represents those observations in the total set of observations $(n)$ where the error of the average estimate is always lower than the error of the first estimate. These observations bring the benefit of averaging estimates (each observation in this subset yields, by definition, a positive number). Here, the second estimate lies in what has been called the "gain range" (Herzog \& Hertwig, 2009). The other three components $\left(n^{2}, n^{3}\right.$, and $n^{4}$ ) are those observations where the error of the average estimate is always higher than the error of the first estimate. ${ }^{6}$ These observations bring the disbenefit of averaging (each

\footnotetext{
${ }^{6}$ Note that there are also observations where the error of the average is identical to the error of the first estimate (i.e., observations where $X^{\mathrm{s}}$ moves towards $X$ while $X^{\mathrm{f}}>X^{\mathrm{s}}=X^{\mathrm{f}}-4\left(X^{\mathrm{f}}-X\right)$ or $X^{\mathrm{f}}<X^{\mathrm{s}}=X^{\mathrm{f}}+4\left(X-X^{\mathrm{f}}\right)$ and observations where $\left.X^{\mathrm{s}}=X^{\mathrm{f}}=X^{\mathrm{a}}\right)$. These observations are not included in the equation because including these does not render any benefit or disbenefit.
} 


\section{DISAGREEING PERSPECTIVES AND ESTIMATION ACCURACY}

observation in these subsets yields, by definition, a negative number). Following Equation 1, averaging first- and second estimates results in an overall improvement when the part that brings benefit (i.e., first component) outweighs the parts that bring disbenefit (i.e., other three components). Likewise, when the parts that brings disbenefit outweigh the part that brings benefit, averaging estimates becomes unbeneficial overall. When looking at each component for the end-scale questions separately per condition (see Table $4 ; n^{c}$ refers to the total set of observations for a particular condition), it becomes clear that the parts that bring disbenefit clearly outweigh the part that brings benefit for the disagree condition (rendering an overall disbenefit of -132.20 in this instance).

\section{[Insert Table 4 about here]}

Table 4. The (dis)benefit of averaging per individual component for all three experimental conditions, Experiment 4 (end-scale questions).

$$
\frac{1}{n^{c}} \sum_{i \in n^{c}}\left(E_{i}^{X^{\mathrm{f}}}-E_{i}^{X^{\mathrm{a}}}\right) \quad \frac{1}{n^{c}} \sum_{i \in n^{1}}\left(E_{i}^{X^{\mathrm{f}}}-E_{i}^{X^{\mathrm{a}}}\right) \quad \frac{1}{n^{c}} \sum_{i \in n^{2}}\left(E_{i}^{X^{\mathrm{f}}}-E_{i}^{X^{\mathrm{a}}}\right) \quad \frac{1}{n^{c}} \sum_{i \in n^{3}}\left(E_{i}^{X^{\mathrm{f}}}-E_{i}^{X^{\mathrm{a}}}\right) \quad \frac{1}{n^{c}} \sum_{i \in n^{4}}\left(E_{i}^{X^{\mathrm{f}}}-E_{i}^{X^{\mathrm{a}}}\right)
$$

$\begin{array}{lccccc}\text { Disagree } & -132.20 & 143.61 & -255.68 & -17.74 & -2.39 \\ \text { Self } & 2.48 & 111.52 & -105.66 & -3.02 & -0.36 \\ \text { Agree } & -6.71 & 91.11 & -95.03 & -2.72 & -0.07\end{array}$

What about the observed higher bracketing rate in the disagreeing-perspective condition for end-scale questions? Following Equation 1, there are two types of brackets. There are brackets - which we refer to as beneficial brackets - where the average estimate is by definition more accurate than the first estimate (e.g., $X=30, X^{\mathrm{f}}=20, X^{\mathrm{s}}=50, X^{\mathrm{a}}=35$ ). Beneficial brackets are observations that follow from $n^{1}$. Unbeneficial brackets, on the other hand, are those observations where the average estimate is by definition less accurate than the first estimate. Unbeneficial brackets are observations that follow from $n^{3}$. These brackets are unbeneficial because the two estimates over-bracket a question's true answer, rendering an 
average estimate that is worse than the first estimate (e.g., $X=30, X^{\mathrm{f}}=20, X^{\mathrm{s}}=80, X^{\mathrm{a}}=50$ ). Although these types of brackets are relatively rare, they occurred more frequently in the disagree condition for the end-scale questions (disagree $=7 \%$; self $=4 \%$; agree $=3 \%$ of the observations in a condition). In sum, while bracketing is indeed a key component when it comes to averaging estimates, it does not by definition render an improvement. That is, averaging estimates becomes unbeneficial once the part that brings benefit (i.e., $n^{1}$ observations including beneficial brackets) is cancelled by observations where the average estimate performs worse than the first estimate (i.e., the $n^{2}-, n^{3}$-, and $n^{4}$-observations).

\section{General Discussion}

Many decisions depend on people's ability to make accurate estimates of unknown quantities and a demonstrated way to improve the accuracy of estimates is to aggregate multiple estimates made by the same person. The potential contained in such an intervention is enormous and a key challenge is to identify strategies that can help improve the accuracy of people's aggregate estimates (Herzog \& Hertwig, 2014a). In this paper, we introduced the following strategy: combine people's first estimate with their second estimate made from the perspective of someone they often disagree with. Across five experiments, we find evidence that such a strategy produces accurate estimates. These results underscore the importance of perspective-taking and disagreement as strategies to improve the accuracy of people's quantitative estimates.

The presented findings laud the benefits of disagreement, a component of people's social interactions that is usually presented as undesirable (Kennedy \& Pronin, 2008; Reeder et al., 2005; Sunstein, 2002). What is particularly interesting is that people obtained more accurate estimates by changing their perspective. It remains to be seen whether taking the perspective of any other - say experts in a particular field - would lead to similar benefits. This might be an important future research direction as our findings demonstrate that taking 
the perspective of others (e.g., agreeing-perspective) might not always render an increase in accuracy as compared to simply making a second guess.

While the inner crowd offered a gain in accuracy, we also identified a situation where it backfired, leading to no improvements or even a worse performance. We found this to be the case when a question's answer was close to the scale's end. Importantly, for participants who employed the disagreeing perspective strategy, the accuracy of their average estimate was much worse than their first estimate for these types of questions. What is particularly interesting is that the propensity of people to move away from the answer when making second estimates is introduced through a feature of the situation rather than some innate bias (Gaertig \& Simmons, in press; Herzog et al., 2019; Müller-Trede, 2011).

Our research also has several limitations. First, the presented evidence is restricted to populations from the US and UK and future work needs to confirm whether these findings hold true in other parts of the world. Second, although combining initial estimates with second estimates made from a disagreeing perspective is beneficial, the presented research remains mute as to whether people would be willing to aggregate both estimates when given the opportunity (Fraundorf \& Benjamin, 2014; Herzog \& Hertwig, 2014b; Müller-Trede, 2011). While prior work indicates that people are more likely to combine their estimates when they actively opposed themselves through dialectical bootstrapping (Herzog \& Hertwig, 2014b), it remains to be seen whether this holds true when the opposition comes from someone with whom they often disagree. People typically view others holding opposing views and opinions less favorably (Iyengar \& Westwood, 2015; Kennedy \& Pronin, 2008; Reeder et al., 2005), potentially undermining their willingness to include the viewpoints of disagreeing others into their own judgments. Future research could address this issue in more detail by testing under what conditions people are willing to combine their estimates with the estimates of disagreeing others to obtain more accurate estimates. 
On a final note, while previous studies often relied on natural processes such as forgetting or the passage of time to improve the accuracy of inner crowds, the present findings report a strategy that is more convenient and time efficient. Similar to other, more active interventions (Herzog \& Hertwig, 2009; Litvinova et al., 2020; Winkler \& Clemen, 2004), taking a disagreeing perspective can likewise be used as a potent strategy when people cannot benefit from the wisdom of an actual crowd. Overall, combining one's first estimate with a second estimate made from the perspective of disagreeing others proves to be a convenient and effective judgment tool. 


\section{References}

Altmejd, A., Dreber, A., Forsell, E., Huber, J., Imai, T., Johannesson, M., Kirchler, M., Nave, G., \& Camerer, C. (2019). Predicting the replicability of social science lab experiments. PLOS ONE, 14(12), e0225826.

Ariely, D., Tung Au, W., Bender, R. H., Budescu, D. V., Dietz, C. B., Gu, H., Wallsten, T. S., \& Zauberman, G. (2000). The effects of averaging subjective probability estimates between and within judges. Journal of Experimental Psychology: Applied, 6(2), 130147.

Baars, J. A., \& Mass, C. F. (2005). Performance of national weather service forecasts compared to operational, consensus, and weighted model output statistics. Weather and Forecasting, 20(6), 1034-1047.

Bates, D., Mächler, M., Bolker, B., \& Walker, S. (2015). Fitting linear mixed-effects models using lme4. Journal of Statistical Software, 67, 1-48.

Batson, C. D., Early, S., \& Salvarani, G. (1997). Perspective taking: Imagining how another feels versus imagining how you would feel. Personality and Social Psychology Bulletin, 23(7), 751-758.

Clemen, R. T. (1989). Combining forecasts: A review and annotated bibliography. International Journal of Forecasting, 5(4), 559-583.

Cohen, J. (1988). Statistical power analysis for the behavioral sciences. $2 \mathrm{~d}$ ed. Hillsdale, N.J.: Lawrence Erlbaum.

de Oliveira, S., \& Nisbett, R. E. (2018). Demographically diverse crowds are typically not much wiser than homogeneous crowds. Proceedings of the National Academy of Sciences of the United States of America, 115(9), 2066-2071.

Epley, N., \& Gilovich, T. (2006). The Anchoring-and-Adjustment Heuristic: Why the Adjustments Are Insufficient. Psychological Science, 17(4), 311-318.

Fraundorf, S. H., \& Benjamin, A. S. (2014). Knowing the crowd within: Metacognitive limits on combining multiple judgments. Journal of memory and language, 71(1), 17-38.

Gaertig, C. \& Simmons, J. P. (in press). The psychology of second guesses. Implications for the wisdom of the inner crowd. Management Science.

Galinsky, A.D., \& Moskowitz, G.B. (2000). Perspective-taking: Decreasing stereotype expression, stereotype accessibility, and ingroup favoritism. Journal of Personality and Social Psychology, 78, 708-724.

Galton, F. (1907). The Ballot-Box. Nature, 75(1952), 509-510. 
Gordon, M., Viganola, D., Dreber, A., Johannesson, M., \& Pfeiffer, T. (2021). Predicting replicability-Analysis of survey and prediction market data from large-scale forecasting projects. PLOS ONE, 16(4), e0248780.

Herzog, S. M., \& Hertwig, R. (2009). The wisdom of many in one mind: Improving individual judgments with dialectical bootstrapping. Psychological Science, 20(2), 231-237.

Herzog, S. M., \& Hertwig, R. (2014a). Harnessing the wisdom of the inner crowd. Trends in Cognitive Sciences, 18(10), 504-506.

Herzog, S. M., \& Hertwig, R. (2014b). Think twice and then: Combining or choosing in dialectical bootstrapping? Journal of Experimental Psychology: Learning, Memory, and Cognition, 40(1), 218-232.

Hoever, I. J., Van Knippenberg, D., Van Ginkel, W., \& Barkema, H. G. (2012). Fostering team creativity: Perspective taking as key to unlocking diversity's potential. Journal of Applied Psychology, 97(5), 982-996.

Hong, L., \& Page, S. E. (2004). Groups of diverse problem solvers can outperform groups of high-ability problem solvers. Proceedings of the National Academy of Sciences, 101(46), 16385-16389.

Hourihan, K. L., \& Benjamin, A. S. (2010). Smaller is better (when sampling from the crowd within): Low memory span individuals benefit more from multiple opportunities for estimation. Journal of Experimental Psychology. Learning, Memory, and Cognition, 36(4), 1068-1074.

Iyengar, S., \& Westwood, S. J. (2015). Fear and loathing across party lines: New evidence on group polarization. American Journal of Political Science, 59, 690-707

Judd, C. M., Westfall, J., \& Kenny, D. A. (2012). Treating stimuli as a random factor in social psychology: A new and comprehensive solution to a pervasive but largely ignored problem. Journal of Personality and Social Psychology, 103(1), 54-69.

Juslin, P., Winman, A., \& Olsson, H. (2000). Naive empiricism and dogmatism in confidence research: A critical examination of the hard-easy effect. Psychological Review, 107(2), 384-396.

Keck, S., \& Tang, W. (2020). Enhancing the wisdom of the crowd with cognitive-process diversity: The benefits of aggregating intuitive and analytical judgments. Psychological Science, 31(10), 1272-1282. 
Kennedy, K. A., \& Pronin, E. (2008). When disagreement gets ugly: Perceptions of bias and the escalation of conflict. Personality and Social Psychology Bulletin, 34(6), 833848.

Kurvers, R. H. J. M., Herzog, S. M., Hertwig, R., Krause, J., Carney, P. A., Bogart, A., Argenziano, G., Zalaudek, I., \& Wolf, M. (2016). Boosting medical diagnostics by pooling independent judgments. Proceedings of the National Academy of Sciences, 113(31), 8777-8782.

Kuznetsova, A., Brockhoff, P. B., \& Christensen, R. H. B. (2017). lmerTest package: Tests in linear mixed effects models. Journal of Statistical Software, 82 (13).

Lakens, D., \& Etz, A. J. (2017). Too true to be bad. Social Psychological and Personality Science, $8(8), 875-881$.

Larrick, R. P., \& Soll, J. B. (2006). Intuitions about combining opinions: Misappreciation of the averaging principle. Management Science, 52(1), 111-127.

Lewis, J., Gaertig, C., \& Simmons, J. P. (2019). Extremeness aversion is a cause of anchoring. Psychological Science, 30(2), 159-173.

Litvinova, A., Herzog, S. M., Kall, A. A., Pleskac, T. J., \& Hertwig, R. (2020). How the "wisdom of the inner crowd" can boost accuracy of confidence judgments. Decision, 7(3), 183-211.

Lord, C.G., Lepper, M.R., \& Preston, E. (1984). Considering the opposite: A corrective strategy for social judgment. Journal of Personality and Social Psychology, 47, 12311243.

Müller-Trede, J. (2011). Repeated judgment sampling: Boundaries. Judgment and Decision Making, 6(4), 12.

Mutz, D. C. (2006). Hearing the other side: Deliberative versus participatory democracy. Cambridge University Press.

Page, S. E. (2008). The Difference: How the Power of Diversity Creates Better Groups, Firms, Schools, and Societies - New Edition. Princeton University Press.

Piaget, J. (1965). The moral judgement of the child. New York: Free Press. (Original work published 1932)

Reeder, G. D., Pryor, J. B., Wohl, M. J. A., \& Griswell, M. L. (2005). On attributing negative motives to others who disagree with our opinions. Personality and Social Psychology Bulletin, 31(11), 1498-1510. 
Soll, J. B., \& Larrick, R. P. (2009). Strategies for revising judgment: How (and how well) people use others' opinions. Journal of Experimental Psychology: Learning, Memory, and Cognition, 35(3), 780-805.

Steegen, S., Dewitte, L., Tuerlinckx, F., \& Vanpaemel, W. (2014). Measuring the crowd within again: A pre-registered replication study. Frontiers in Psychology, 5. 786-794.

Stroop, J. R. (1932). Is the judgment of the group better than that of the average member of the group? Journal of Experimental Psychology, 15(5), 550-562. https://doi.org/10.1037/h0070482

Sunstein, C. R. (2002). The law of group polarization. Journal of Political Philosophy, 10(2), 175-195.

Surowiecki, J. (2005). The wisdom of crowds. New York: Doubleday Press.

Tversky, A., \& Kahneman, D. (1992). Advances in prospect theory: Cumulative representation of uncertainty. Journal of Risk and Uncertainty, 5(4), 297-323.

Van Dolder, D., \& Van den Assem, M. J. (2018). The wisdom of the inner crowd in three large natural experiments. Nature Human Behaviour, 2(1), 21-26.

Vul, E., \& Pashler, H. (2008). Measuring the crowd within: Probabilistic representations within individuals. Psychological Science, 19(7), 645-647.

Wallsten, T.S., Budescu, D.V., Erev, I., \& Diederich A. (1997). Evaluating and combining subjective probability estimates. Journal of Behavioral Decision Making, 10, 243268.

Winkler, R. L., \& Clemen, R. T. (2004). Multiple experts vs. multiple methods: Combining correlation assessments. Decision Analysis, 1(3), 167-176. 